\title{
LA SITUACIÓN DE LAS MUJERES EN EL EMPLEO PÚBLICO: ANÁLISIS Y PROPUESTAS
}

\section{The situation of women in public employment: Analysis and proposals}

\author{
ALBA SORIANO ARNANZ \\ Universitat de València \\ alba.soriano@uv.es
}

Cómo citar/Citation

Soriano Arnanz, A. (2021).

La situación de las mujeres en el empleo público: análisis y propuestas.

lgualdadES, 4, 87-121.

doi: https://doi.org/10.18042/cepc/lgdES.4.03

(Recepción: 04/02/2021; aceptación tras revisión:19/04/2021; publicación: 30/06/2021)

\section{Resumen}

La desigualdad de género continúa siendo una realidad persistente que se ve reflejada en todos los ámbitos de la sociedad. Si bien las instituciones del sector público han realizado un notable esfuerzo por eliminar dicho problema, lo cierto es que al tratarse de instituciones construidas sobre valores tradicionalmente masculinos siguen reproduciendo los estereotipos de género que suponen la base de la discriminación estructural hacia las mujeres. El presente trabajo analiza la situación de desigualdad entre mujeres y hombres en el empleo público centrándose, en especial, en la situación de segregación horizontal y vertical que todavía hoy persiste en este ámbito y presentando una serie de propuestas encaminadas a la consecución de la igualdad de mujeres y hombres en el empleo público.

\section{Palabras clave}

Género; igualdad; discriminación; segregación; empleo público; sector público. 


\begin{abstract}
Gender inequality continues to be a persistent reality reflected in all areas of society. The public sector has made significant efforts in order to eliminate this problem. However, given the fact that these institutions have been built upon traditional male values, they still reproduce gender stereotypes, which are at the base of structural discrimination against women. This paper analyses the situation of inequality between men and women in the public sector. It particularly focuses on the phenomenon of gender segregation in public employment and presents a series of proposals aimed towards achieving gender equality in public administrations.
\end{abstract}

\title{
Keywords
} istration.

Gender; equality; discrimination; segregation; public employment; public admin- 


\section{SUMARIO}

I. INTRODUCCIÓN. II. ROLES DE GÉNERO Y DIVISIÓN SEXUAL DEL TRABAJO. III. LAS MUJERES EN EL ÁMBITO LABORAL: SECTOR PÚBLICO Y SECTOR PRIVADO: 1. Presencia de mujeres en el sector público y privado: ámbito nacional y comparado. 2. Temporalidad en el sector público y privado. 3. Brecha salarial. IV. LA NECESIDAD Y OBLIGACIÓN DE PERSEGUIR LA IGUALDAD PLENA DE MUJERES Y HOMBRES EN EL EMPLEO PÚBLICO. V. LA SEGREGACIÓN VERTICAL Y HORIZONTAL POR RAZÓN DE GÉNERO EN EL EMPLEO PÚBLICO: 1. Profesiones feminizadas: educación no universitaria y sanidad. 2. Profesiones masculinizadas: fuerzas y cuerpos de seguridad del Estado. 3. Acceso por convocatorias específicas: docencia universitaria. 4. Acceso por convocatorias generales: Administración General del Estado. VI. DISCUSIÓN: 1. La perpetuación de los roles de género y la importancia de la forma de selección del personal. 2. Los resultados de las políticas de igualdad derivadas de la LOI. 3. La necesidad de más y mejor información y diagnósticos. 4. Las recientes reformas normativas en materia de igualdad en el empleo y su limitada aplicación al sector público. VII. PROPUESTAS: 1. Mejora de los sistemas de recogida de datos y transparencia para la equiparación de las condiciones laborales de profesiones masculinizadas y feminizadas. 2. Estrategias formativas y de concienciación. 3. Adopción de medidas concretas en la contratación, promoción y organización del personal empleado público. VIII. ALGUNAS CONCLUSIONES Y RETOS. BIBLIOGRAFÍA.

\section{INTRODUCCIÓN}

Las normas de contratación de personal empleado público y el control al que el sector público está sometido facilitan que las relaciones de género sean más igualitarias en el seno de las organizaciones públicas que en el sector privado (Mora y Ruíz-Castillo, 2004: 401). Es por ello que el análisis desde la perspectiva de género del empleo público es en no pocas ocasiones relegado a un segundo plano al entenderse que existen otros ámbitos en los que las relaciones de desigualdad de género son mucho más acuciantes y que, por tanto, requieren de una mayor atención. 
Si bien es cierto que los niveles de desigualdad entre mujeres y hombres en el sector público son menores que en el sector privado, la igualdad en el empleo público dista de ser una realidad efectiva en todas sus dimensiones (De Luxán Meléndez, 2018; Tello-Sánchez, 2020). Es por ello que, en la medida que lo que se pretende es la consecución de una sociedad igualitaria, resulta todavía hoy imprescindible analizar, detectar y encontrar soluciones a aquellos aspectos de las organizaciones públicas que continúan produciendo situaciones desiguales por razón de género.

El presente trabajo pretende ofrecer una visión general de la situación y, en algunos casos, también de la evolución de las mujeres en el empleo público. Se parte de la teoría feminista y de cómo esta explica la existencia de unos roles de género que se reproducen en el mercado de trabajo. A continuación se examina la presencia de mujeres y hombres en el sector privado y público, así como los niveles de temporalidad y la brecha salarial, con el objetivo de demostrar la desigualdad que todavía hoy existe entre las y los empleados públicos. Se señalan los motivos por los cuales es necesario construir un sector público igualitario y se apunta a la segregación vertical y horizontal como una de las causas y síntomas de la discriminación indirecta que afecta a las empleadas públicas. Por último, se realizan unas breves reflexiones sobre la situación actual, las políticas adoptadas y el marco normativo y una serie de propuestas encaminadas a la consecución de la igualdad en el sector público.

\section{ROLES DE GÉNERO Y DIVISIÓN SEXUAL DEL TRABAJO}

La construcción patriarcal de la sociedad y de todos los poderes públicos halla su base en la formación del contrato sexual (Pateman, 1988), en virtud del cual las mujeres quedaban relegadas a la ocupación del espacio privado, constituido por el ámbito doméstico, al tiempo que los hombres ocupaban el espacio público, es decir, la esfera del mercado laboral y de la toma de decisiones; en definitiva, el ámbito del poder (Cobo, 1995).

Esta distribución de funciones y espacios se encuentra, a su vez, directamente relacionada con la asignación de una serie de valores diferenciados a mujeres y a hombres (Rodríguez Ruiz y Rubio Marín, 2009: 1181). Así, al tiempo que a la mujer se le atribuyen, por ejemplo, los valores relativos al cuidado, la solidaridad, la cooperación, la empatía, dependencia y la irracionalidad, entre otros, a los hombres se les atribuyen los valores de agresividad, competitividad, racionalidad, objetividad e independencia (Fernández Ruíz-Gálvez, 2003: 160-161).

Tal como apuntan Rodríguez Ruiz y Rubio Marín (2007: 143-144) «el tránsito al Estado democrático no puso en cuestión el contrato social como 
mito fundacional del Estado, ni cuestionó el pacto entre los sexos en que, de forma estructural y necesaria, el contrato social a su vez se apoya». Es por ello que los roles de género, como cualidades aprendidas que diferencian a ambos sexos, siguen estando presentes en la sociedad de manera que, como norma general, se tiende a educar a hombres y mujeres en valores distintos (Marrades Puig, 2020: 387-388). Dado que la construcción del poder se viene realizando sobre este modelo en el que son los hombres los que ocupan la esfera pública, los valores integrados en las organizaciones de poder tanto del sector público como del sector privado (empresas, instituciones públicas, etc.) han sido, tradicionalmente, aquellos asociados con la masculinidad (Salazar Benítez, 2019: 60). Pero es que, además, esta división sexual entre esferas pública y privada se construye como una estructura jerárquica en la que la esfera privada y los valores adscritos a esta se encuentran en una posición de subordinación con respecto al ámbito público y los valores y roles asociados a este (Fernández Ruíz-Gálvez, 2003:160-161).

La subordinación de los valores femeninos a los masculinos derivó en que la entrada de las mujeres en el ámbito público no fuese acompañada del acceso de los valores asociados a estas a dicho espacio ni de los masculinos a la esfera privada (Fernández de Castro y Díaz, 2016: 3). Así, teniendo en cuenta la difícil mutabilidad de las normas y valores propios de las sociedades y, en especial, de las grandes organizaciones burocráticas, las cuales han sido construidas sobre la base de los valores típicamente masculinos, sigue siendo posible apreciar en el ámbito laboral, tanto en el sector público como en el privado, una clara jerarquización en virtud de la cual se otorga una menor importancia a aquello considerado como femenino.

Por tanto, no se trata exclusivamente de que el acceso de las mujeres al ámbito público no viniese acompañado de la incorporación de los valores femeninos a dicha esfera sino que se produjo, y se continúa produciendo, una segregación en la clase de empleos desempeñados por hombres y por mujeres (Sánchez-Mira, 2017: 60). Existen en el imaginario colectivo una serie de profesiones consideradas como femeninas al estar relacionadas con el cuidado. Estas profesiones son desarrolladas en su mayoría por mujeres (Hernández Martín et al., 2004: 109; Connell, 2006: 841) y su desempeño por hombres puede incluso llegar a verse, aún hoy en día, como extraño.

En sentido contrario, existen también determinadas profesiones cuyo desempeño es automáticamente identificado con lo masculino como, por ejemplo, las profesiones más técnicas y, fundamentalmente, las que precisan el empleo de la fuerza. Es más, esta segregación horizontal en el empleo reproduce la jerarquización de los valores femeninos y masculinos, recibiendo los empleos asociados a las mujeres una menor valoración social (traducida, a su vez, en una menor remuneración) que aquellos cuyo desempeño se entiende 
más vinculado con valores masculinos (Fernández Ruíz-Gálvez, 2003: 166; Hernández Martín et al., 2004: 108).

Continuando con esta idea, la mayor valoración social que reciben sobre todo las profesiones científicas y técnicas en comparación con otras ramas del conocimiento como las humanidades deriva en que las mujeres tiendan a ocupar en mayor medida estos ámbitos. Así, no nos encontramos únicamente con que las mujeres ocupan las profesiones más vinculadas al rol del cuidado u otras que comprenden, por ejemplo, tareas de secretaría, sino también, y de manera general, aquellas que son menos valoradas socialmente (Mosteiro García, 1997: 310).

Por otra parte, no hay que olvidar que la construcción del poder desde su ocupación por hombres y, por tanto, tomando como base los valores masculinos, genera asimismo una segregación vertical en el empleo. La segregación vertical implica que los hombres ocupan aquellos puestos más cercanos al poder incluso en profesiones feminizadas. Así, al tiempo que es más complicado ascender para las mujeres en profesiones masculinizadas (Charles, 2003: 269) e incluso sentirse cómodas en su entorno laboral (Chen, 2015: $10)$, los hombres que se dedican a profesiones feminizadas tienden a sobresalir con respecto a sus compañeras y encajar a la perfección (Simpson, 2004: 352).

Esta realidad tiene como consecuencia las diferencias retributivas entre mujeres y hombres y una mayor precarización de los empleos típicamente ocupados por mujeres (Sánchez-Mira, 2017). Por supuesto, existen otros posibles marcos de análisis que tratan de explicar las diferencias en la situación entre hombres y mujeres en el mercado laboral. Desde la perspectiva de la oferta, se ha argumentado que mujeres y hombres difieren en sus preferencias con respecto al tipo de profesión que quieren desempeñar (Vega Catena, 2014: 58). Desde la perspectiva de la demanda, algunos economistas se han referido a las diferencias en productividad entre mujeres y hombres (Domínguez Martín y Sánchez-Sánchez, 2007: 145-146). Sin embargo, consideramos dichos marcos de análisis poco satisfactorios por cuanto que no tienen en cuenta la discriminación estructural que históricamente y todavía hoy afecta a las mujeres y que explica su situación en el mercado de trabajo.

En este sentido, cabe destacar que, como han demostrado múltiples estudios, la clase de competencias y aptitudes a las que se suele dar una mayor valoración en el mercado de trabajo son aquellas que se corresponden con los valores típicamente masculinos (Lousada Arochena, 2018; Sánchez-Mira, 2017; Oelz et al., 2013; OIT, 2015), que son aprendidos por los hombres al ser socializados en ellos (Marrades Puig, 2020: 387-388). Asimismo, con respecto a la diferencia en las preferencias de mujeres y hombres en relación con las ramas de empleo que tienden a ocupar, así como con las decisiones vitales tomadas en relación con la reducción de jornadas o paralización temporal 
de la vida laboral, no parece suficiente limitarse a señalar dichas diferencias sin buscar donde se encuentra su origen. Es por ello que consideramos que el marco de análisis feminista tiene una capacidad explicativa mucho más completa a la hora de evaluar las diferencias entre mujeres y hombres en el ámbito laboral (Sánchez-Mira, 2017: 87).

\section{LAS MUJERES EN EL ÁMBITO LABORAL: SECTOR PÚBLICO Y SECTOR PRIVADO}

\section{PRESENCIA DE MUJERES EN EL SECTOR PÚBLICO Y PRIVADO: ÁMBITO NACIONAL Y COMPARADO}

La incorporación de las mujeres al mercado laboral en España tuvo lugar, fundamentalmente, a partir del último tercio del siglo xx y, en especial, desde el inicio de la transición hacia la democracia (Millán Vázquez de la Torre et al., 2015). Si echamos la mirada atrás hasta 1976, las mujeres constituían alrededor del $28 \%$ de las personas ocupadas en España, contando con una presencia similar tanto en el sector público como en el sector privado (De Luxán Meléndez, 2018: 289-290). Desde ese momento, su presencia en ambos sectores fue aumentando progresivamente hasta superar en número desde el año 2003 en el sector público a los trabajadores masculinos (id.; Encuesta de Población Activa; Millán Vázquez de la Torre et al., 2015).

Según los datos de la Encuesta de Población Activa (en adelante, EPA) del cuarto trimestre de 2020, las mujeres representan el 45,88\% de personas ocupadas en España. El 56,84\% de las personas empleadas en el sector público son mujeres, pero su presencia en el sector privado disminuye hasta el $43,56 \%{ }^{1}$.

De los datos expuestos es posible concluir que el sector público ha favorecido y continúa favoreciendo la presencia de las mujeres en el mercado laboral. Sin embargo, cabe destacar que España todavía se sitúa por debajo de la media OCDE $(59,6 \%$ en 2017) y a una distancia muy significativa de los países escandinavos en la proporción de mujeres ocupadas en el sector público. Así, en Finlandia, Suecia, Noruega y Dinamarca las mujeres

\footnotetext{
1 En las series temporales con datos extraídos de la EPA los gráficos incluyen los datos desde 2002, dado el cambio de metodología e información contenido que se dio en dicho momento. En cualquier caso, consideramos que dicha serie temporal muestra suficientemente la evolución de la situación de las mujeres en el sector público y privado, así como los posibles efectos que hayan podido tener los cambios normativos en materia de igualdad de mujeres y hombres.
} 
Gráfico 1. Ocupados por sexo y tipo de sector (público o privado). Evolución 2002-2020

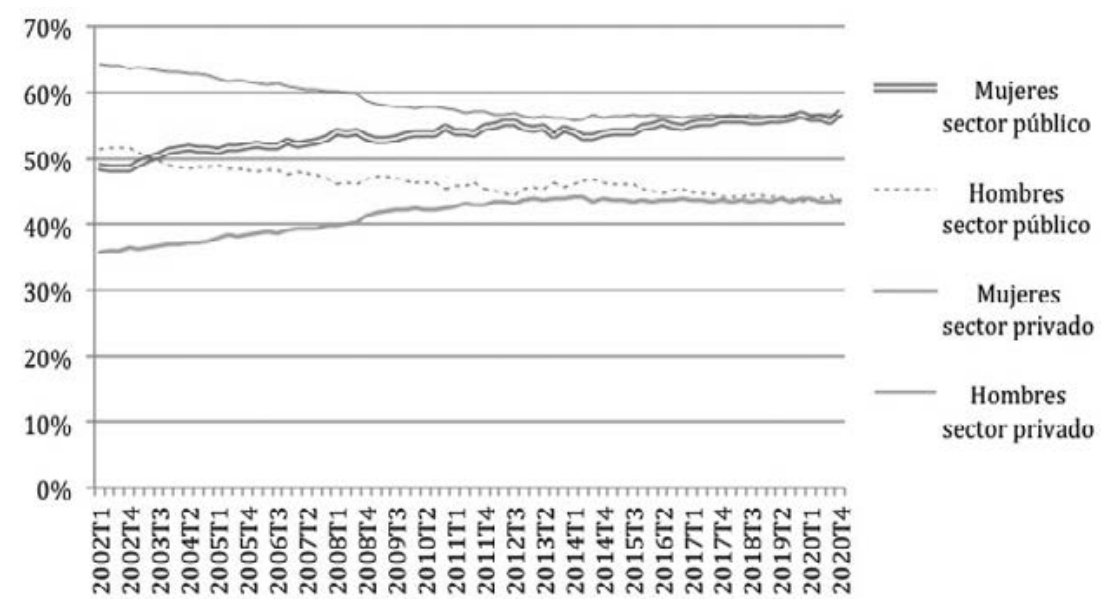

Fuente: elaboración propia a partir de datos extraídos del INE (EPA, 4T 2020).

representan de un $69 \%$ a un 71,3\% del personal empleado público. España se sitúa también por detrás de países como Italia o Francia (OCDE, 2019: 88-89).

Esta realidad no implica necesariamente que deba aumentarse la proporción de mujeres en el empleo público en España pues, si bien es cierto que todos los países mencionados tienen un menor índice de desigualdad de género que España según el indicador desarrollado por el Programa de las Naciones Unidas para el Desarrollo (PNUD, 2020), también lo es que, en el caso de Italia, el índice europeo de igualdad de género sitúa a este país por detrás de España (EIGE, 2020) y que muchos de los Estados que superan a España en la proporción de mujeres empleadas públicas se sitúan, tanto en el índice europeo como en el de Naciones Unidas, por detrás de España en igualdad de género a nivel global (EIGE, 2020; PNUD, 2020).

Asimismo, tal como indica el propio informe de la OCDE del que se han extraído los datos relativos a la proporción de mujeres en el empleo público, la mayor presencia de mujeres en este ámbito se debe, en gran medida, al hecho de que muchas de las profesiones desarrolladas en el sector público se corresponden con profesiones típicamente feminizadas. Así sucede, en particular, en el caso del profesorado infantil y de primaria y la enfermería (OCDE, 2019: 88).

En este sentido, el hecho de que las mujeres sean mayoría entre el personal empleado público no es óbice para que continúen produciéndose situaciones de desigualdad entre mujeres y hombres reflejados, entre otras cuestiones, en 
Gráfico 2. Proporción de mujeres en el empleo público en paises de la OCDE

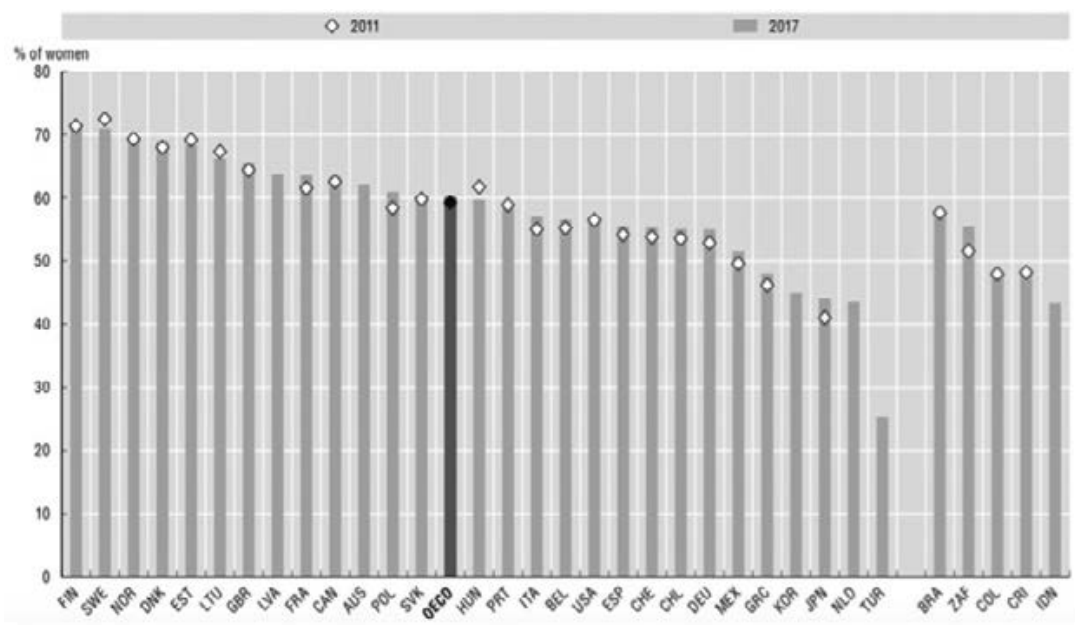

Fuente: OCDE (2019:89).

la mayor proporción de mujeres en empleos temporales del sector público o en la brecha salarial.

\section{TEMPORALIDAD EN EL SECTOR PÚBLICO Y PRIVADO}

Uno de los grandes problemas del empleo público es el elevado nivel de temporalidad en el trabajo del sector público en España que, sobre todo, se acentuó como consecuencia de los efectos de la crisis de 2008 y que afecta, de manera desproporcionada a mujeres (De Luxán Meléndez, 2020; González López, 2019).

Las diferencias por sexo en contratos temporales pueden medirse de dos maneras. En primer lugar, respecto del total de mujeres asalariadas podemos hacer referencia a qué porcentaje tiene contratos temporales y comparar dicha cifra con la proporción de hombres asalariados con contratos temporales respecto del total de hombres asalariados. En segundo lugar, podemos calcular, del total de contratos temporales realizados, la proporción ocupada por hombres y la proporción ocupada por mujeres.

Tomando en consideración a la primera medida, cabe destacar que en el cuarto trimestre de 2020 del total de mujeres asalariadas en el sector público un $35 \%$ tenían contratos temporales; esta cifra ha ido aumentando desde 2002, fecha en la que eran un $27 \%$ de las mujeres asalariadas del sector público las que contaban con contratos temporales. Dicha cifra contrasta con 
Gráfico 3. Proporción de personas asalariadas con contratos temporales por sexo y sector (público o privado). Evolución 2002-2020

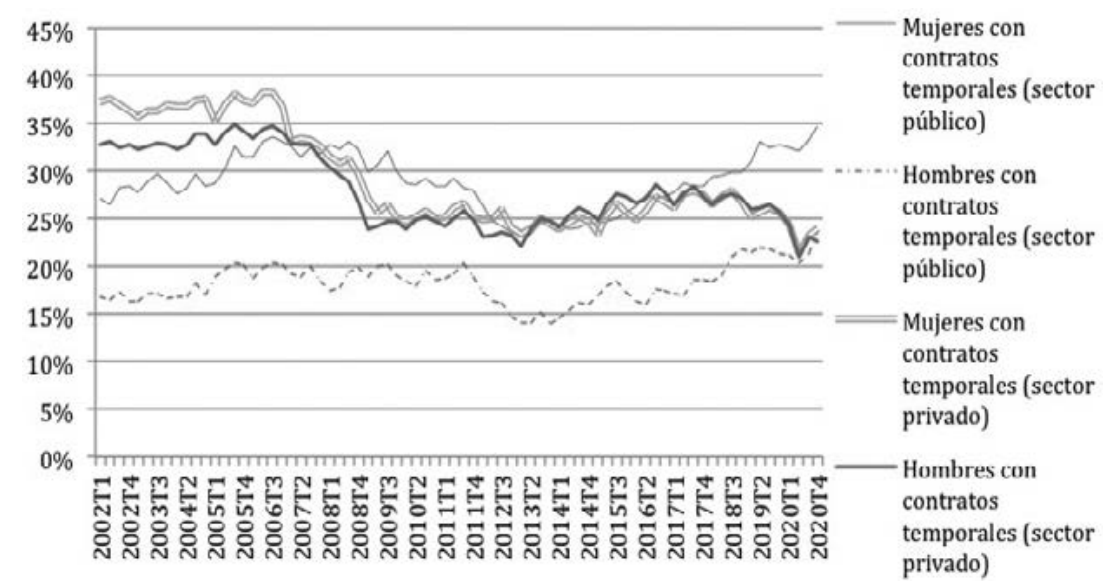

Fuente: elaboración propia a partir de datos extraídos del INE (EPA, 4T 2020).

la proporción de mujeres con contratos temporales en el sector privado, que en el cuarto trimestre de 2020 se situaba en torno al 24\%, habiendo descendido en 13 puntos desde 2002.

Con respecto a los hombres, la temporalidad masculina en el sector público ha aumentado también, pero en menor medida que en el caso de las mujeres. Así, del total de hombres asalariados en el sector público, un $17 \%$ tenían contratos de carácter temporal en 2002, cifra que aumentó hasta un $24 \%$ en 2020. Con respecto al sector privado, de un $33 \%$ de hombres asalariados con contratos temporales en el primer trimestre de 2002, la cifra ha descendido progresivamente hasta situarse en un $23 \%$ en el cuarto trimestre de 2020.

Con respecto a la segunda posible medida de desigualdad en la contratación temporal de mujeres y hombres en el sector público, del total de contratos temporales en el primer trimestre de 2002, un $61 \%$ correspondían a mujeres y un 39\% a hombres; en el caso de las mujeres, dicha cifra ha aumentado hasta un $66 \%$ en el cuarto trimestre de 2020 y se ha reducido para los hombres (34\%).

En el sector privado, en el primer trimestre de 2002 la distribución de contratos temporales era de $40 \%$ en mujeres frente al $60 \%$ en hombres. Esta diferencia ha ido cerrándose, de manera que en el cuarto trimestre de 2020 la distribución era de $47 \%$ en mujeres y de $53 \%$ en hombres. 
De los datos expuestos, a nivel general se aprecia una tendencia a la reducción de la temporalidad en el sector privado, aunque dicha tendencia parece haberse estabilizado desde la crisis de 2008. Sin embargo, la reducción general en temporalidad también ha venido acompañada de un aumento en la proporción de mujeres con contratos temporales. En caso de estabilizarse la distribución, esta realidad no sería excesivamente problemática. Ahora bien, si la tendencia actual persiste, sí podría darse una situación de desproporción en la representación de mujeres con contratos temporales.

En el sector público apreciamos una tendencia al aumento de la temporalidad $^{2}$. Consideramos conveniente resaltar que desde las instituciones de la UE recientemente se ha llamado la atención a España por la elevada tasa de temporalidad en el empleo público (Maqueda, 2021). Esta situación afecta, sobre todo, a las mujeres. Así como la diferencia entre la cantidad de hombres y mujeres con contratos temporales se ha ido equilibrando en el caso del sector privado, esta no ha hecho sino aumentar en el caso del sector público. Cabe asimismo destacar que la distribución de contratos indefinidos en el sector público se encuentra bastante equilibrada, al constituir las mujeres un 53\% del personal empleado público con contrato indefinido.

No solo resulta preocupante la elevada temporalidad en el empleo público y el hecho de que esta va en aumento sino, en especial, la elevada feminización del empleo temporal en el sector público. Esta situación se debe a la mayor temporalidad del trabajo en aquellos sectores más feminizados (De Luxán Meléndez, 2020), pero también a que el aumento de la temporalidad en el sector público implica que, en no pocos ámbitos de este, la forma normal de acceso es a través de puestos de carácter temporal (interinos) y que las mujeres que acceden de esta forma más adelante tienen mayores dificultades para compatibilizar los estudios con las tareas de cuidado que siguen asumiendo mayoritariamente. Cabe dibujar un paralelismo de esta situación con la menor presencia de mujeres en el acceso por promoción interna, que se justifica por idénticas razones (Ministerio de Política Territorial y Función Pública, 2020b: 31).

2 Si bien no se pretenden analizar los efectos de la crisis de la COVID-19 en la temporalidad en el empleo por exceder dicha cuestión del ámbito de análisis de este trabajo, sí cabe resaltar que el aumento en la temporalidad en el empleo público que ha tenido lugar en el último año parece haber afectado de manera bastante igualitaria a ambos sexos, al tiempo que las mujeres han resultado más perjudicadas por la destrucción de empleo temporal en el sector público. 


\section{BRECHA SALARIAL}

Hay diferentes formas de medir la brecha salarial. Dado que lo que se pretende es ofrecer una visión general de la situación de las mujeres en el empleo público, a continuación se ofrecen los datos relativos a la brecha salarial no ajustada. La brecha salarial no ajustada es aquella que mide la diferencia retributiva entre mujeres y hombres sin atender a características que pueden explicar la diferencia, como pueden ser el nivel de estudios o la experiencia profesional. Esta medida sirve para demostrar las diferencias, en general, entre mujeres y hombres en el mercado laboral y contribuye a evidenciar la existencia de una estructura sociolaboral que sitúa a las mujeres en una posición de desventaja (Carretero García, 2019: 13-14).

Gráfico 4. Brecha salarial de género por sector (público o privado). Evolución 2010-2020

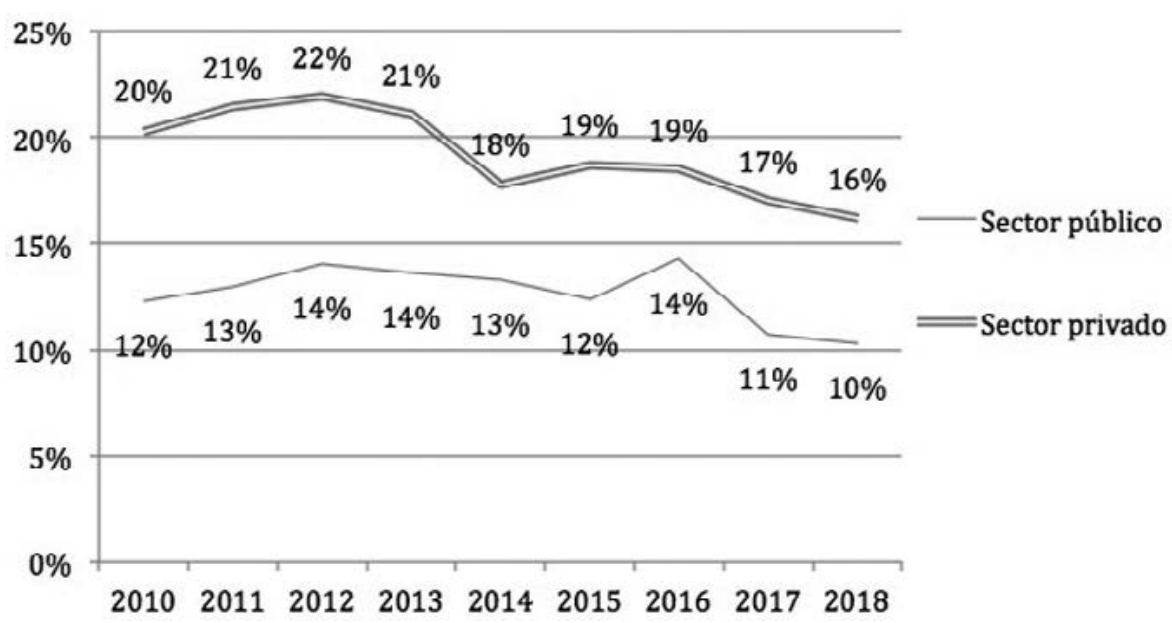

Fuente: elaboración propia a partir de datos extraídos de Eurostat y la Encuesta Cuatrienal de Estructura Salarial (INE, 2018).

Aunque la brecha salarial por razón de género es menor en el sector público que en el sector privado, esta todavía persiste. Así, de acuerdo con Eurostat en 2018, última fecha para la que se dispone de datos, la brecha salarial de género sin ajustar se situaba en un $10,3 \%$ en el sector público y en un $16 \%$ en el sector privado. 


\section{LA NECESIDAD Y OBLIGACIÓN DE PERSEGUIR LA IGUALDAD PLENA DE MUJERES Y HOMBRES EN EL EMPLEO PÚBLICO}

Conviene recordar que las organizaciones del sector público, como poderes públicos del Estado, tienen encomendada, de conformidad con lo dispuesto en el art. 9.2 CE, la promoción de la igualdad y la remoción de obstáculos para la consecución de este objetivo. También el art. $14 \mathrm{CE}$ establece la igualdad ante la ley y la prohibición de discriminación por razón de sexo y el art. 23.2 CE fija el derecho a la igualdad en el acceso a las funciones y cargos públicos.

La propia normativa en materia de empleo público, en desarrollo del marco constitucional referido, incorpora la igualdad como principio al que deben de atender los procesos de selección y promoción de personal, mandato que no solo se desprende de los preceptos antecitados, sino también del propio art. 103 CE. El Real Decreto Legislativo 5/2015, de 30 de octubre, por el que se aprueba el texto refundido de la Ley del Estatuto Básico del Empleado Público (en adelante, TREBEP), establece en su art. 10.2 que el personal funcionario será seleccionado de conformidad con los principios de «igualdad, mérito y capacidad». Asimismo, en su art. 14. c) se indica que todas las personas empleadas públicas tienen derecho «a la progresión en la carrera profesional y promoción interna según principios constitucionales de igualdad, mérito y capacidad mediante la implantación de sistemas objetivos y transparentes de evaluación».

Más allá del deber de respetar la igualdad como principio y derecho fundamental reconocido en la Constitución Española y en la normativa en materia de empleo público, la consecución de un sector público que respete plenamente la igualdad de mujeres y hombres se deriva de dos otros factores.

En primer lugar, no debemos olvidar que las condiciones de acceso y laborales propias del sector público han facilitado el acceso y permanencia de las mujeres en el mercado laboral. En la medida que el empleo público ha supuesto y supone un factor de aumento de la presencia de mujeres en el mercado laboral y que, como ya se ha reconocido, las desigualdades entre mujeres y hombres en el sector público son menores que en el sector privado, resulta necesario reconocer que el empleo público supone un factor de igualación entre mujeres y hombres en la sociedad. Por consiguiente, resulta esencial profundizar en la consecución de la igualdad plena entre el personal del sector público para así continuar avanzando en la mejora de la situación de las mujeres a nivel general en la sociedad.

En segundo lugar, es necesario que los poderes públicos asuman e integren la igualdad como valor propio tal como, de manera progresiva, han ido reconociendo los órganos legislativos al incorporar y desarrollar dicho 
mandato en el ordenamiento jurídico español. La Ley Orgánica 3/2007, de 22 de marzo, para la igualdad efectiva de mujeres y hombres (en adelante LOI) dedica su título $\mathrm{V}$ al principio de igualdad en el empleo público. Cabe fundamentalmente destacar el art. 51 de dicha norma, dado que este establece una serie de principios generales y transversales de actuación positiva de las instituciones públicas para la conquista de la igualdad de mujeres y hombres en el empleo público (Tur Ausina, 2008: 412-420).

Teniendo en cuenta que las Administraciones públicas desarrollan su actividad bajo la supervisión del Gobierno correspondiente (Santamaría Pastor, 2016: 24), en principio podría bastar con que se cumpliesen las funciones que tienen encomendadas en materia de igualdad de género sin que fuese necesario que interiorizasen la igualdad como valor propio. Este ha sido el entendimiento tradicional, profundamente insatisfactorio por poco exigente y en el fondo no acorde a los valores constitucionales. Dicha visión ha sido ya superada por casi todos los actores sociales y políticos, tanto en España como en nuestro entorno comparado, al comprenderse que las Administraciones públicas, como instituciones, se caracterizan por una serie de elementos de los que se deriva la necesidad de que la igualdad se integre como valor propio de aquellas.

Las instituciones públicas son elementos duraderos de las sociedades en las que se encuentran, por lo que modificar sus valores y normas internas es un proceso difícil. Al tener unos valores fuertemente asentados las instituciones constriñen y moldean el comportamiento de sus agentes, sin perjuicio de que estos puedan influir y dirigir en cierta medida el funcionamiento de aquellas (Alfama Guillén, 2015: 266-267; Mahoney y Thelen, 2010: 4).

Así pues, las instituciones del sector público han sido construidas sobre unos valores patriarcales, valores que por las características propias de las instituciones son difícilmente mutables, y dichas organizaciones son tanto el reflejo como el ejemplo de la sociedad en la que se encuentran (Alfama Guillén, 2015: 267-268). Dado que no nos hallamos ante unas organizaciones neutras, sino que tienen un claro sesgo de género (Mahoney y Thelen, 2010: 580), no es posible entender la consecución de la igualdad de género que las instituciones públicas tienen encomendada sin que, tanto estas como su personal, integren y asuman dicho objetivo como valor propio, sobre todo si se tiene en cuenta que el sesgo de género de las instituciones afecta a las políticas implementadas y las normas desarrolladas por ellas (ibid.: 582).

Asumiendo estas insuficiencias, el actual marco normativo refleja no solo la importancia de que todas las instituciones públicas persigan la consecución de la igualdad en sus relaciones con la ciudadanía, sino que también integren la igualdad en su seno. Así, la LOI expresa claramente este objetivo al hablar en su exposición de motivos de la necesidad «del compromiso de los sujetos 
públicos» con la igualdad y lo materializa, entre otros elementos, al establecer en el capítulo ii del título II una serie de normas relativas a la «acción administrativa para la igualdad»y, en lo que realmente ocupa al presente trabajo, dedica el título $\mathrm{V}$ al «principio de igualdad en el empleo público».

Teniendo en cuenta lo anteriormente expuesto, resulta necesario analizar la segregación sexual en el empleo público al constituir esta realidad uno de los elementos que demuestran la forma en que los estereotipos de género siguen presentes en el sector público e influyen en la discriminación estructural e indirecta sufrida por las mujeres. Asimismo, el análisis de la segregación vertical y horizontal por razón de género en el sector público también puede contribuir a detectar aquellos ámbitos en los que se debe actuar con el objetivo de construir unas instituciones públicas más igualitarias.

\section{LA SEGREGACIÓN VERTICAL Y HORIZONTAL POR RAZÓN DE GÉNERO EN EL EMPLEO PÚBLICO}

Tal como indica el III Plan para la igualdad de género en la Administración General del Estado y en los organismos públicos vinculados o dependientes de ella (Ministerio de Política Territorial y Función Pública, 2020b: 5):

En las Administraciones Públicas se reproducen las mismas dinámicas, roles, costumbres y estereotipos de género que en cualquier otra organización y que en otros ámbitos de nuestra sociedad. Estos roles y estereotipos son la base, en gran medida, de las desigualdades entre mujeres y hombres. Los sesgos de género, las conductas sexistas, y las barreras invisibles (propias y ajenas) influyen, por ejemplo, en la promoción profesional — techo de cristal—, en una segregación o representación desigual según qué ocupaciones profesionales, o en la división tradicional de los roles de cuidado, y explican diferentes situaciones de discriminación directas e indirectas.

El sector público se caracteriza por la persistencia de una segregación sexual del trabajo tanto a nivel horizontal como vertical, aunque esta aparezca en menor medida que en el ámbito privado (Hernández Martín et al., 2004: 107). La ocupación de áreas profesionales diferenciadas implica, entre otras cuestiones, la devaluación de los trabajos a los que acceden mayoritariamente mujeres y trae consigo una reducción proporcional en la remuneración percibida en dichos ámbitos (segregación horizontal), así como la ocupación por parte de mujeres de las áreas dentro de un sector en las que se perciben salarios más bajos (segregación vertical) (Bettio y Verashchagina, 2009: 65). 
Las próximas páginas ofrecen datos relativos a las diferencias entre mujeres y hombres en diferentes ámbitos del sector público. Los ámbitos escogidos son: educación no universitaria, sanidad, fuerzas y cuerpos de seguridad del Estado, universidades y Administración General del Estado (en adelante, AGE).

Con respecto a los tres primeros ámbitos, estos muestran, principalmente, la existencia de una segregación horizontal en el empleo público. La educación no universitaria y la sanidad son sectores eminentemente feminizados al estar relacionados con el cuidado en los que, sin embargo, sigue existiendo una segregación vertical. Las fuerzas y cuerpos de seguridad del Estado emplean principalmente a hombres al desarrollarse en este ámbito una profesión cercanamente asociada a estereotipos de la masculinidad.

La universidad y la AGE se escogen por la diferencia en la forma de acceso y desarrollo profesional y el diferente efecto que estos sistemas tienen en la presencia de mujeres. El sistema de acceso de las universidades públicas españolas, más individualizado y adaptado al puesto de trabajo concreto y, en este sentido, hasta cierto punto, más cercano a los sistemas de selección del sector privado, favorece una mayor presencia de hombres. Por su parte, el sistema de acceso por convocatorias generales propio de la AGE favorece el incremento de la presencia de mujeres. En todo caso, se aprecia la existencia de segregación vertical, aunque algo menor en el caso de la AGE, marcada también por la segregación horizontal que supone la mayor presencia de mujeres en puestos con funciones de secretaría (categoría C2).

\section{PROFESIONES FEMINIZADAS: EDUCACIÓN NO UNIVERSITARIA Y SANIDAD}

Como ya se ha indicado, la atribución del rol del cuidado a las mujeres implica la existencia de una serie de profesiones que se entienden como femeninas y que son, en su mayoría, ejercidas por mujeres. La docencia no universitaria, en la que el alumnado es generalmente menor de edad y, por consiguiente, precisa que se le preste una mayor atención, es uno de esos ámbitos del mercado de trabajo principalmente ocupados por mujeres al entenderse socialmente que el rol de cuidadoras atribuido a estas las hace inherentemente capaces de ser responsables de los primeros años de formación de las personas (Hernández Martín et al., 2004: 110). En este sentido, el porcentaje de mujeres docentes en los centros de educación de régimen general públicos, es decir, aquellos centros que imparten enseñanzas en preescolar, primaria, 
secundaria, bachillerato o formación profesional, se situaba alrededor del $72 \%$ en el curso $2018 / 2019^{3}$.

Particularmente llamativo es el hecho de que a medida que avanzan los cursos y el alumnado requiere menos cuidados y es más independiente por ir avanzando en edad, se reduce la diferencia entre profesorado masculino y femenino. Así, al tiempo que el porcentaje de mujeres dentro de la categoría de maestra o maestro es de casi un $82 \%$, se reduce a un $61 \%$ en el caso del profesorado de educación secundaria, y a algo menos del $50 \%$ para el profesorado de educación secundaria con la categoría de catedrática o catedrático. Asimismo, las mujeres representan un $46 \%$ del profesorado técnico de formación profesional.

Cabe asimismo resaltar que la representación de mujeres en la educación no universitaria ha aumentado en nueve puntos desde el curso 1999/2000. Dicho aumento se refleja de igual manera en las categorías de maestra o maestro y de profesorado de educación secundaria, categorías en las que las mujeres representaban en el curso 1999/2000 un $73 \%$ y $52 \%$ del profesorado respectivamente. El aumento más significativo se da en la presencia de mujeres entre el profesorado técnico de formación profesional, al constituir estas únicamente un 34\% de dicho personal en el curso 1999/2000. La proporción de mujeres catedráticas también ha aumentado desde el $44 \%$ que representaban en el momento en que se incorporó esta figura en el curso 2007/2008.

Los datos expuestos en este último párrafo reflejan tanto una segregación horizontal como vertical. La segregación horizontal se produce por la reducción de mujeres en aquellos sectores de docencia que no requieren el ejercicio de un gran rol de cuidado, como el profesorado de secundaria y el profesorado técnico de formación profesional. La segregación vertical se produce, fundamentalmente, en los ascensos a la categoría de catedrática o catedrático de educación secundaria, que no se corresponde con la presencia de mujeres en los cuerpos de profesorado de secundaria. Dicha segregación vertical también es apreciable en los porcentajes de mujeres y hombres que se dedican a la enseñanza secundaria, dado que el hecho de que en dicha enseñanza se requiera menor ejercicio del cuidado implica una mayor valoración social de dicha profesión.

En todo caso, cabe destacar la perpetuación de los roles de género, no ya solo porque las mujeres constituyan la inmensa mayoría del personal docente

3 Todos los datos contenidos en este epígrafe se extraen del Ministerio de Educación. Los últimos datos definitivos disponibles son los correspondientes al curso 2018/2019: Enseñanzas no universitarias/ Estadística del profesorado/Curso 2018-2019. Disponible en: http://estadisticas.mecd.gob.es/. 
en la educación no universitaria, sino también porque dicha proporción ha ido en aumento durante los últimos veinte años. Esta situación se puede deber a que la creciente feminización del alumnado de las universidades españolas ha tendido a perpetuar los roles de género al elegir las mujeres carreras universitarias que se corresponden con los estereotipos tradicionalmente asignados a ellas. En este sentido, la proporción de mujeres en carreras universitarias (en universidades públicas y privadas) relacionadas con la educación ha aumentado desde un $72 \%$ en el curso 1999/2000 hasta un $78 \%$ en el curso 2019/2020.

Con respecto al personal sanitario cabe indicar, en primer lugar, que, si bien existen abundantes datos relativos al personal sanitario colegiado, no es fácil encontrar datos que desagreguen el personal público sanitario por sexo. En cualquier caso, igual que en la docencia no universitaria, hay una amplia mayoría de mujeres en el sector sanitario, fundamentalmente en las ocupaciones más próximas al cuidado, es decir, enfermería, auxiliar de enfermería y matronas.

Algunas comunidades autónomas, como la valenciana, sí disponen de datos al haber establecido planes de igualdad muy completos en el ámbito sanitario. En este sentido, en la Comunidad Valenciana el porcentaje de mujeres enfermeras en la sanidad pública se sitúa en un $82,36 \%$ y el porcentaje de mujeres auxiliares de enfermería y comadronas en un 95,78\% y 95,69\%, respectivamente (Comissió d'Igualtat de la Conselleria de Sanitat Universal i Salut Pública, 2016: 20). A pesar de que las mujeres representan alrededor de un $75 \%$ del personal sanitario y no sanitario (ibid.: 19), constituyen únicamente un $43,59 \%$ del personal directivo sanitario y un $48,9 \%$ del personal directivo no sanitario (ibid:: 22).

Cifras muy similares se repiten en la sanidad pública a lo largo de todo el territorio español (Ministerio de Sanidad, 2009: 80). Los últimos datos disponibles a nivel nacional con respecto a los porcentajes de hombres y mujeres que ejercen la enfermería en el sector público son de 2009. Sin embargo, teniendo en cuenta la proporción de mujeres y hombres colegiados, es previsible que no hayan variado en exceso. En dicho momento, la proporción de mujeres que ejercían la enfermería en el sector público se situaba en casi un $85 \%$. En la actualidad, según los datos del INE, las mujeres representan un $84 \%$ entre los y las profesionales de enfermería colegiadas.

Asimismo, cabe destacar que no parece que se esté cumpliendo el mandato contenido en el art. 27.3.e) que establece el deber de asegurar «La presencia equilibrada de mujeres y hombres en los puestos directivos y de responsabilidad profesional del conjunto del Sistema Nacional de Salud». Si bien no existen datos completos al respecto, existen estudios que fijan la proporción de mujeres directoras gerentes de hospitales públicos alrededor 
en un $25 \%$ del total (Marcos, 2018). Asimismo, resulta destacable la brecha salarial existente en el sector sanitario y servicios sociales, muy por encima de la media en el empleo público, al situarse en torno al $28 \%$ en 2018 (FESPUGT, 2021: 16).

\section{PROFESIONES MASCULINIZADAS: FUERZAS Y CUERPOS DE SEGURIDAD DEL ESTADO}

El porcentaje de mujeres que forman parte del personal de las fuerzas y cuerpos de seguridad del Estado se sitúa en torno al 11\% (Ministerio de Política Territorial y Función Pública, 2020a: 10), cifra que ha aumentado en tan solo cinco puntos desde $2007^{4}$ (Ministerio de Administraciones Públicas, 2007: 41). La escasa presencia de mujeres es producto de que, como norma, se asocie el ejercicio de estas profesiones a un tipo de desempeño masculino como consecuencia del uso de la fuerza que implican (Butler et al., 2003: 299). Sin duda, este es el ámbito en el que más patente se hace la segregación por razón de género dentro del sector público.

Con el objetivo de conocer y mejorar la situación de las mujeres en la Policía Nacional y la Guardia Civil, así como de fomentar el acceso de las mujeres a dichos cuerpos, se creó en el año 2007 el Observatorio de la Mujer en las Fuerzas y Cuerpos de la Seguridad del Estado. Sin embargo, tras una primera reunión, la actividad de dicho observatorio no continuó. La Dirección General de la Policía Nacional también creó en 2018 la Oficina Nacional para la Igualdad de Género dentro de la Policía que sí ha desarrollado distintas estrategias formativas y documentos con el objetivo de promover la igualdad de mujeres y hombres en la Policía Nacional.

\section{ACCESO POR CONVOCATORIAS ESPECÍFICAS: DOCENCIA UNIVERSITARIA}

Con respecto a la docencia universitaria cabe indicar que el porcentaje de mujeres es menor al de hombres, ya que supone un $42 \%$ del personal docente investigador de los centros públicos para el curso 2018/20195.

\footnotetext{
4 Este fue el primer año en el que los datos del Registro Central de Personal se desagregaron por sexo.

5 Todos los datos contenidos en este epígrafe se extraen del Ministerio de Educación. Los últimos datos disponibles se refieren al curso 2019/2020. Estadistica de Personal de las Universidades: personal docente e investigador (PDI). Curso 2017/2018Final del formulario.
} 
Distinguiendo por área de conocimiento, se evidencia que hay determinadas áreas, como la Enfermería o algunas filologías, en las que existen una amplia mayoría de mujeres, situándose el porcentaje de personal docente investigador (PDI) en torno al $70 \%$, con el notable ejemplo del área de Filología Francesa, en la que únicamente hay un $22 \%$ de hombres. En el ámbito de la Enfermería, llama enormemente la atención que no haya ni una sola catedrática de universidad y que las mujeres constituyan tan solo un $41 \%$ del personal catedrático de escuela universitaria.

Por su parte, en las áreas más técnicas, sobre todo en las ingenierías, el porcentaje de mujeres entre el personal docente investigador se reduce notablemente, situándose alrededor del $11 \%$ en Ingeniería Aeroespacial o Construcciones Navales.

A nivel general cabe destacar que la rama de la enseñanza en la que más diferencias por razón de género se producen es en Ingeniería y Arquitectura, en las que tan solo un $23 \%$ del PDI son mujeres. Esta situación se debe principalmente a la menor presencia de mujeres en los estudios universitarios en estas áreas, situación que se da como resultado de la creencia consciente o inconsciente de que las titulaciones relacionadas con la ciencia y la tecnología, fundamentalmente en áreas como la Informática, no constituyen espacios apropiados para mujeres (Fernández García et al., 2014: 89).

Con respecto a las categorías profesionales ocupadas por hombres y mujeres en este ámbito hay un mayor número de mujeres en las categorías no funcionariales (ayudantes doctoras y contratadas doctoras), pero más hombres entre el personal funcionario (profesores titulares y catedráticos). De esta manera, alrededor del $50 \%$ de las ayudantes doctoras y del personal contratado doctor son mujeres. Sin embargo, las mujeres únicamente constituyen el $41 \%$ del profesorado titular y el $23 \%$ de las y los catedráticos.

Como es evidente, esta segregación vertical puede tener que ver, en parte, con la incorporación más tardía de las mujeres a los estudios universitarios, por lo que cabría esperar la reversión de esta situación en las próximas décadas. Sin embargo, las mujeres son mayoría en los estudios universitarios desde el curso 1986-1987, por lo que si esta fuese la única o incluso principal causa por la que hay menos mujeres entre el profesorado universitario, la presencia de mujeres y hombres ya debería haberse igualado.

Asimismo, si esa supuesta tendencia a la igualación fuese real, debería apreciarse en la proporción de mujeres catedráticas por tramos de edad. Sin embargo, la proporción de mujeres catedráticas está en torno al $26 \%$ en el tramo de edad de 40-49 años, en el $28 \%$ en el tramo de 50-59 y en el $25 \%$ en el tramo de 60-64. Sí existe un aumento considerable en la proporción de mujeres en estos tramos con respecto al tramo de edad de 65 o más años, al alcanzar las mujeres solo un $19 \%$ de este segmento. Por otra parte, 
también cabe destacar que, si bien es cierto que los datos referentes al número de catedráticos en el tramo 34-39 no son muy relevantes (puesto que solo hay cuatro), sí conviene resaltar que todos ellos son hombres.

Por tanto, las resistencias a la igualación de situaciones entre mujeres y hombres, sobre todo entre catedráticos y catedráticas, no se deben atribuir a una cuestión puramente generacional. El primer elemento lo constituyen las situaciones vitales propias de las mujeres, como la maternidad y, en general, la asignación del rol del cuidado, que pueden llegar a paralizar su carrera profesional durante años (Hernández Martín et al., 2004: 141). El segundo elemento es el propio machismo estructural y los valores que les son asignados, que dificulta el acceso de las mujeres a categorías profesionales más elevadas. En este sentido, resulta relevante apuntar, aunque no profundicemos en ello por razones de espacio, a los sexenios y, en particular, a los recientemente lanzados sexenios de transferencia como otro factor de desigualdad en la docencia universitaria (ANECA, 2020; López Díaz et al., 2020).

\section{ACCESO POR CONVOCATORIAS GENERALES: ADMINISTRACIÓN GENERAL DEL ESTADO 6}

Las mujeres constituyen el $51 \%$ de la AGE (Ministerio de Política Territorial y Función Pública, 2020b: 13). La presencia de mujeres aumenta considerablemente en las categorías laborales temporales al constituir un 69\% del personal funcionario interino y el $57,3 \%$ del personal temporal (ibid.: 14-15).

También en la AGE se aprecia segregación horizontal por ministerios y cuerpos. Con respecto al reparto del personal funcionario por ministerios cabe indicar que hay una mayoría de mujeres en todos los ministerios excepto Defensa, Economía, Fomento e Interior. Con respecto a los cuerpos, las mujeres son minoría en aquellos relacionados con la ingeniería o la informática, entre otros (Ministerio de Política Territorial y Función Pública, 2020b: 21).

La presencia de mujeres y hombres es equilibrada en los grupos A1 (el $47 \%$ de mujeres), A2 (el $53 \%$ de mujeres) y C1 (44\% de mujeres). Sin embargo, al pasar al grupo C2 de la Administración del Estado se observa que el porcentaje de mujeres aumenta hasta alcanzar el $68 \%$ y se reduce enormemente en el caso de las agrupaciones profesionales, al constituir las mujeres

6 Los datos expuestos en este apartado se extraen del III Plan para la igualdad de género en la Administración General del Estado y en los organismos públicos vinculados o dependientes de ella. 
únicamente el $25 \%$ de esta clase de personal (Ministerio de Política Territorial y Función Pública, 2020a: 26). Con respecto a los grupos del personal laboral, si bien la distribución no es totalmente equilibrada en todos ellos, no se aprecia segregación vertical.

Como datos relevantes para mostrar la segregación vertical en la AGE nos centramos en el grupo A1 por ser este el de mayor nivel y al que más puestos de libre designación corresponden. Resulta destacable que la presencia de mujeres se reduzca a un $42 \%$ en el nivel 30 y a un $40 \%$ en el nivel 29 , a pesar de constituir estas un $47 \%$ del personal del grupo A1 (Ministerio de Política Territorial y Función Pública, 2020b: 35). Asimismo, las mujeres ocupan el $42 \%$ de los puestos de libre designación del grupo A1, cinco puntos por debajo de su proporción en el total del grupo.

La presencia de mujeres en los niveles más elevados ha aumentado en los últimos diez años, ya que estas constituían un $33 \%$ del personal de grupo A1 de nivel 30 y un $31 \%$ del nivel 29 en el año 2009. Con respecto a los puestos de libre designación, la proporción de mujeres del grupo A1 en estos puestos era tan solo del $34 \%$. La proporción de mujeres en el grupo A1 en aquel momento era del $41 \%$.

Según los datos contenidos en el III Plan de Igualdad, la proporción de mujeres en los altos cargos de la AGE en enero de 2019 era de un $31 \%$ (ibid.: 37). Sin embargo, de conformidad con los datos del Instituto de las Mujeres, este porcentaje era del $42 \%$ en 2019 y del 43\% 2020 (Instituto de las Mujeres, 2020b). Esta diferencia se explica, en parte, porque el Plan de Igualdad tiene en cuenta la proporción de embajadoras y delegadas del Gobierno. Sin embargo, eliminando esas dos figuras, la proporción de mujeres en altos cargos según el III Plan de Igualdad de la AGE sería de $36 \%$.

\section{DISCUSIÓN}

\section{LA PERPETUACIÓN DE LOS ROLES DE GÉNERO Y LA IMPORTANCIA DE LA FORMA DE SELECCIÓN DEL PERSONAL}

En primer lugar, con respecto a los datos analizados, conviene destacar que el sector público, a pesar de caracterizarse por una mayor igualdad en el empleo que el sector privado, todavía se encuentra lejos de la igualdad plena entre mujeres y hombres. Entre otras cuestiones, resulta necesario destacar el hecho de que la temporalidad afecta en mayor medida a las mujeres empleadas públicas que a los hombres. Asimismo, también debemos referirnos a la brecha salarial que en muchos casos puede en parte derivarse de la valoración de 
criterios aparentemente objetivos y que, sin embargo, perjudican a las mujeres, como ocurre en la evaluación de los sexenios del profesorado universitario.

La segregación horizontal del trabajo todavía persiste en el empleo público de manera que aquellos sectores en los que se desarrollan actividades relacionadas principalmente con valores asociados a lo femenino o a lo masculino se encuentran, a su vez, principalmente ocupados por mujeres u hombres respectivamente. Asimismo, cabe destacar que, en áreas feminizadas, la proporción de mujeres ha ido en aumento. De esta manera se contribuye a perpetuar la asignación del rol del cuidado a las mujeres y los diferentes estereotipos relativos a hombres y mujeres.

La segregación vertical, que se encuentra directamente relacionada con las diferencias retributivas entre mujeres y hombres, se aprecia en prácticamente todos los ámbitos de la Administración, incluso en aquellos más feminizados. Así, la proporción de mujeres en puestos de dirección o en las escalas superiores de la carrera profesional es inferior al porcentaje que representan dentro del grupo e incluso, en algunas ocasiones, inferior a la proporción de hombres en dichos puestos. Esta reducción de mujeres en puestos con mayor poder de decisión, dirección y gestión tiene que ver con la perpetuación de los roles y estereotipos de género y se acentúa dada la forma de selección de las personas que ocupan dichos puestos de trabajo, al existir mayor margen de discrecionalidad en dichas decisiones.

La relevancia del sistema de forma de acceso también se hace patente si comparamos, por ejemplo, la presencia de mujeres en la AGE y en las universidades españolas. Así, aunque sería necesario profundizar en la relación de causalidad entre forma de acceso y presencia de mujeres, todo parece indicar que el acceso por convocatorias generales facilita el acceso de más mujeres. En sentido contrario, los sistemas de acceso por convocatorias específicas, en las que el puesto de trabajo de destino se encuentra previamente individualizado, favorece a los hombres (De Luxán Meléndez, 2018: 302). En general, aquellas formas de selección más similares a las propias del sector privado parecen perjudicar a las mujeres en mayor medida, lo que a su vez demuestra que la igualdad de mujeres y hombres no se ha interiorizado como valor propio de las instituciones públicas españolas y que la discriminación indirecta sufrida por las mujeres en el ámbito laboral en general se refleja también en el empleo público.

\section{LOS RESULTADOS DE LAS POLÍTICAS DE IGUALDAD DERIVADAS DE LA LOI}

La concreción de las políticas de igualdad se realiza, principalmente, a través de los planes de igualdad. Estos planes contribuyen a la realización de un diagnóstico de las diferencias entre hombres y mujeres en las organizaciones 
para las que se realizan. La disposición adicional séptima del TREBEP obliga a las Administraciones públicas a establecer planes de igualdad.

Los planes y políticas de igualdad adoptados por las Administraciones públicas en España se han desarrollado en un contexto de creciente reconocimiento de la desigualdad histórica y estructural sufrida por las mujeres y de impulso por combatirla desde las instituciones, pero también a muchos otros niveles de la sociedad. En general, el marco normativo en materia de igualdad y las políticas públicas desarrolladas a partir de este tienen una innegable importancia en la mejora de la situación de las mujeres en general y, en concreto en el empleo público como ámbito que nos ocupa (Ruiloba Núñez y Navarro González, 2020: 361).

Sin embargo, la desaparición de la segregación horizontal y vertical que todavía persiste en el sector público no parece avanzar al ritmo y, en algunos casos, ni siquiera en el sentido que sería deseable. En este sentido, cabe destacar que en algunas ocasiones la adopción de políticas de igualdad no viene acompañada de un verdadero compromiso por parte de las instituciones públicas (Pastor Gosálbez et al., 2020: 163-164). Así, por ejemplo, a partir de la crisis del 2008, cuyos efectos en el sector público se notaron especialmente desde 2011, el compromiso con la igualdad de mujeres y hombres pasó a un segundo plano y se vio especialmente perjudicado por las políticas de austeridad (Espinosa y Bustelo, 2019: 154; Paleo y Alonso, 2014).

\section{LA NECESIDAD DE MÁS Y MEJOR INFORMACIÓN Y DIAGNÓSTICOS}

Una cuestión problemática de la regulación relativa a los planes de igualdad de las Administraciones públicas es que no existe obligación de realizar planes específicos por sector de actividad dentro de cada Administración. Si bien es cierto que no resulta necesario realizar diagnósticos para cada uno de los grupos de actividad de las Administraciones públicas, hay determinados sectores que presentan determinadas características que hacen recomendable abordar, de manera específica, las diferencias en el empleo entre hombres y mujeres. Así, por ejemplo, sectores como la sanidad, en la que la mayor proporción de mujeres empleadas no impide la existencia de una segregación vertical y brecha salarial a favor de los hombres, o las fuerzas y cuerpos de seguridad del Estado, en los que la presencia de mujeres es todavía, a día de hoy, casi anecdótica.

En todo caso, conviene resaltar que los planes de igualdad realizados por las Administraciones públicas, como norma general, se caracterizan por ser muy completos y realizar diagnósticos con bastante profundidad de las diferencias entre mujeres y hombres. Simplemente se pretende señalar que el desarrollo de diagnósticos específicos y explicativos de las diferencias por áreas 
de actividad y también entre ellas aportaría una información más completa acerca de la situación de las mujeres en el sector público.

Existen abundantes datos relativos a la situación de las mujeres en el mercado laboral y, en concreto, en el empleo público en España. Ahora bien, ello no quiere decir que no exista margen de mejora en la recogida y exposición de datos, así como en la elaboración de diagnósticos de la situación de las mujeres en el empleo público, sobre todo en relación con la distribución retributiva.

\section{LAS RECIENTES REFORMAS NORMATIVAS EN MATERIA DE IGUALDAD EN EL EMPLEO Y SU LIMITADA APLICACIÓN AL SECTOR PÚBLICO}

En 2019, se aprobó el «Real Decreto-ley 6/2019, de 1 de marzo, de medidas urgentes para garantía de la igualdad de trato y de oportunidades entre mujeres y hombres en el empleo y la ocupación» (en adelante, RDL 6/2019), que reconoce los discretos resultados de las medidas de naturaleza promocional y de fomento derivadas de la aprobación de la LOI. El RDL 6/2019 introduce modificaciones en la regulación de los planes de igualdad, en relación con el control de la discriminación retributiva y en materia de conciliación, cuidados y protección frente al despido discriminatorio. La aplicación de esta norma al personal funcionario se limita a las disposiciones relativas a los permisos para el cuidado de menores (maternidad, paternidad, adopción, etc.). Con la aprobación del RDL 6/2019 se pretenden resolver algunas de las insuficiencias contenidas en la normativa existente en materia de igualdad. Entre las nuevas medidas adoptadas cabe destacar el detalle con el que se regula el contenido de los planes de igualdad, así como la obligación de inscripción obligatoria de estos en el Registro de planes de igualdad y depósito de medidas y protocolos para prevenir el acoso sexual y por razón de sexo.

En desarrollo del RDL 6/2019 se ha aprobado el "Real Decreto 901/2020, de 13 de octubre, por el que se regulan los planes de igualdad y su registro y se modifica el Real Decreto 713/2010, de 28 de mayo, sobre registro y depósito de convenios y acuerdos colectivos de trabajo» (en adelante RD 901/2020); sin embargo, esta norma no resulta de aplicación al sector público (Instituto de las Mujeres, 2020a). Consideramos conveniente resaltar, sobre todo, el contenido del anexo del RD 901/2020 que, de manera bastante amplia, desarrolla todos los elementos que han de constar en el diagnóstico de desigualdad. Esta regulación debería, en principio, contribuir al incremento de la efectividad de los planes de igualdad como mecanismos para señalar y modificar aquellos elementos propios de la estructura organizativa de las empresas que contribuyen a perpetuar la desigualdad entre hombres y mujeres en el ámbito laboral. 
La correcta fiscalización del contenido de los planes de igualdad desarrollados en el marco del RD 901/2020 debería derivar en la progresiva desaparición de los planes genéricos y poco adaptados a las organizaciones específicas para las que se realizan que tan comunes han sido desde la entrada en vigor de la LOI (Ballester Pastor, 2019: 20). Asimismo, la pormenorizada relación del contenido de los diagnósticos de desigualdad debería también ayudar a superar algunas de las justificadas críticas realizadas a la genérica regulación del contenido obligatorio de los planes de igualdad que efectúa el RDL 6/2019 (ibid.: 20-21).

También en desarrollo del RDL 6/2019 se ha aprobado el «Real Decreto 902/2020, de 13 de octubre, de igualdad retributiva entre mujeres y hombres» (en adelante, RD 902/2020), que resulta de aplicación el personal laboral de las Administraciones públicas. Esta norma establece las normas relativas a la transparencia en las retribuciones, registro retributivo y auditorías retributivas que deben cumplir todas las empresas con más de cincuenta personas empleadas.

En conjunto, los RD 901/2020 y 902/2020 son herramientas enormemente útiles para reducir la segregación sexual en el trabajo y equiparar la situación laboral de mujeres y hombres y, sin desmerecer la enorme relevancia que la equiparación de permisos para el cuidado tiene, sería conveniente que el sector público integrase también las normas referentes a los planes de igualdad y en relación con la igualdad retributiva para la totalidad de su personal. En este sentido, cabe valorar muy positivamente el hecho de que en el III Plan de Igualdad de la AGE se indique que el análisis de la brecha salarial se realizará, al parecer, en relación con todo su personal, de conformidad con los mandatos del RD 902/2020 (Ministerio de Política Territorial y Función Pública, 2020b: 96). Sin embargo, es de gran relevancia que se tenga en cuenta que ambas normas (RD 901/2020 y 902/2020) son complementarias y, para su efectividad, deben ser aplicadas conjuntamente.

\section{PROPUESTAS}

1. MEJORA DE LOS SISTEMAS DE RECOGIDA DE DATOS Y TRANSPARENCIA PARA LA EQUIPARACIÓN DE LAS CONDICIONES LABORALES DE PROFESIONES MASCULINIZADAS Y FEMINIZADAS

Uno de los problemas fundamentales de la segregación sexual horizontal en el empleo es la jerarquización de las profesiones consideradas femeninas y masculinas, situándose estas por encima de aquellas en su valoración social $y$, en ocasiones, también en la remuneración económica percibida (Sevilla 
Merino et al., 2017: 37). En este sentido cabe recordar, por todas, las SSTC 145/1991, 22/1994, STJUE de 28 de febrero de 2013 dictada en el Asunto 427/11 y STS 1908/2014 en las que se establece la existencia de supuestos de discriminación indirecta al haberse otorgado mayor valor $\mathrm{y}$, por ende, compensación, a aquellas categorías profesionales más masculinizadas que a otras principalmente ocupadas por mujeres. Así, por ejemplo, las normas contenidas en los RD 901/2020 y 902/2020, de aplicarse plenamente a todo el sector público, pueden resultar útiles a la hora de evaluar las diferentes categorías profesionales en el empleo público y las diferencias retributivas.

También es esencial evaluar muchos otros elementos, como la compensación por rendimiento (sexenios), que tienden a configurarse de forma que perjudican a las mujeres. Es por ello que, en la línea del estudio desarrollado por la Asociación de Mujeres Científicas y Tecnólogas (López Díaz et al., 2020) deben analizarse las razones por las que las mujeres logran acceder en menor medida a esta clase de mejoras profesionales, participan menos en las convocatorias de promoción interna o tienden a ocupar puestos de carácter temporal en mayor medida que los hombres.

\section{ESTRATEGIAS FORMATIVAS Y DE CONCIENCIACIÓN}

Con el objetivo de atajar la segregación vertical en el empleo público ha de tenerse en cuenta que algunas de las medidas implementadas para facilitar la vida laboral de las mujeres adaptándose a sus situaciones vitales pueden terminar por perjudicar la progresión de sus carreras profesionales. Por ejemplo, la posibilidad de reducir la jornada de trabajo o pedir excedencias por tener personas a cargo, a la que se acogen en su mayoría mujeres, si bien evita la expulsión de dichas mujeres del mercado laboral tras los años dedicados a los cuidados, dificulta a la postre su ascenso profesional (Blau y Kahn, 2013: 255; Cain, 2015).

Por consiguiente, debe fomentarse que un mayor número de hombres opten por los permisos que facilitan la corresponsabilidad familiar. Para ello, deben realizarse estrategias formativas de concienciación para intentar, entre otras cuestiones, eliminar de la conciencia social el hecho de que optar por esta clase de permisos denota una falta de interés en el trabajo. Cabe tomar como ejemplo el sistema establecido en Reino Unido con el objetivo de fomentar que los hombres soliciten el permiso de paternidad compartido con sus compañeras, proporcionando información a aquellos acerca de su derecho a solicitar dicho permiso, ofrecerles asesoramiento personalizado en esta materia y publicitar los casos de personal de alta dirección que ha solicitado el permiso compartido de paternidad (UK Government, 2019). 
También cabe tener en cuenta con respecto a la segregación vertical que una de las principales razones por las que las mujeres acceden en menor medida a puestos de mayor poder o responsabilidad, incluso en sectores más feminizados, es la construcción de los roles de género en virtud de la cual las mujeres no son educadas para ocupar puestos de poder. Para ello, tal como ya se ha hecho en países como Italia, deberían impulsarse programas de formación y fomento de la ocupación por mujeres de puestos de responsabilidad (OCDE, 2014: 49).

Asimismo, estrategias formativas para concienciar y formar en igualdad al personal son esenciales sobre todo en aquellos sectores más masculinizados que en muchas ocasiones resultan hostiles para las mujeres empleadas en ellos (Chen, 2015: 10). Para asegurar la consecución de los objetivos de los cursos de formación en materia de igualdad es necesario que todo el personal reciba dichos cursos o que, al menos, haya paridad en la composición del alumnado. Asimismo, no basta que se realicen formaciones en materia de igualdad para considerar que dichos objetivos han sido cumplidos pues es necesario realizar evaluaciones relativas a su efectividad.

Cabe destacar que algunas de estas acciones ya han sido realizadas en algunos cuerpos de las Administraciones públicas (MHFP, 2018: 19-23) y también se incluyen en el III Plan de Igualdad de la AGE (Ministerio de Política Territorial y Función Pública, 2020b: 72-84), pero deben también extenderse a todo el sector público. En todo caso, la equiparación de permisos derivada del RDL 6/2019 sin duda redundará en un aumento de la corresponsabilidad.

\section{ADOPCIÓN DE MEDIDAS CONCRETAS EN LA CONTRATACIÓN, PROMOCIÓN Y ORGANIZACIÓN DEL PERSONAL EMPLEADO PÚBLICO}

Los mecanismos de contratación, promoción y organización del personal empleado público juegan un papel fundamental, tanto directo como indirecto, en la producción de la segregación horizontal y vertical en el sector público, así como en las condiciones laborales de las empleadas públicas. Estos mecanismos pueden ayudar a reducir la segregación horizontal y vertical fomentando la incorporación de mujeres a aquellos ámbitos en los que tengan menor presencia (Connell, 2006: 837) y mejorar aquellos aspectos, como la elevada temporalidad del sector público, que esencialmente perjudican a las mujeres. Empezando con este último aspecto, es de vital importancia repensar el sistema actual de provisión de puestos de trabajo con el objetivo de reducir la temporalidad en el sector público.

La obligación de promocionar la contratación de mujeres ha sido introducida, con diferentes variantes, en los ordenamientos jurídicos de algunos 
Estados. Por ejemplo, en Suecia existe la obligación, tanto para las y los empleadores del sector público como del privado, de fomentar la contratación del sexo infrarrepresentado en el área de la que formen parte, tal como establece sección novena del capítulo tercero de la ley sueca sobre discriminación de 2008.

Otra razón por la que las propuestas realizadas en el presente apartado son necesarias es que algunas de las medidas puestas en marcha para facilitar la vida laboral de las mujeres adaptándose a sus situaciones vitales puede terminar por perjudicar la progresión de sus carreras profesionales (Martínez García, 2017: 1-31). Asimismo, no puede olvidarse la reticencia por parte de los hombres, incluso tras la introducción de estrategias formativas y de promoción de la igualdad y de la corresponsabilidad, a adoptar el rol del cuidado tradicionalmente asignado a las mujeres. En este sentido cabe destacar que actualmente, y tras doce años de vigencia de la LOI y quince de la Ley Integral contra la Violencia de Género, en España, las mujeres continúan representando un $96 \%$ del total de personas con reducción de jornada por cuidado de menores o de personas adultas enfermas, incapacitadas o mayores (INE, 2019).

Como solución temporal a esta situación, que inevitablemente todavía tardará en equilibrarse, en el cómputo de méritos para la selección y promoción del personal empleado público debería fijarse un coeficiente corrector, aumentando la puntuación de las mujeres en función del número de descendientes, así como del número de personas a cargo. Asimismo, no debería descontarse del tiempo trabajado los periodos en los que se haya disfrutado de reducciones de jornada laboral o permisos por cuidado de personas dependientes.

Además, podrían fijarse cuotas para los puestos de libre designación o, como mínimo, la obligación de que un determinado porcentaje de las personas candidatas en cualquier convocatoria a esta clase de puestos sean mujeres. Ahora bien, esta medida debería ser aplicada con extremada cautela dado que podría derivar en que únicamente se propusiesen a mujeres para determinados puestos, pero con el objetivo final de nombrar a hombres. Es por ello que debería comprobarse que todas las personas propuestas se encuentren debidamente capacitadas para ocupar el puesto de trabajo en cuestión y que la persona seleccionada sea efectivamente la más idónea. Aunque existe la posibilidad de que este sistema no aportase los resultados esperados, al menos serviría para visibilizar a las mujeres preparadas para ocupar puestos de trabajo con mayores responsabilidades.

Otra posible medida sería la incorporación de un sistema de acceso directo a puestos de dirección en el empleo público tras un periodo formativo específico al que pudiesen acceder las personas que obtuviesen mejores resultados en sus estudios universitarios (Boix y Soriano, 2020). Teniendo en cuenta que las mujeres tienden a obtener mejores resultados académicos, la 
implementación de esta medida probablemente resultaría en que un mayor número de mujeres accediese a puestos de dirección. Este sistema de acceso directo debería ser complementado con un curso de formación especializada en el ámbito correspondiente.

Finalmente, dentro de la contratación y organización del empleo público también se propone el diseño de un programa de prácticas cuyo objetivo principal fuese reforzar la presencia y formación de mujeres en ámbitos en los que estas tienen menor presencia en el sector público (id.).

\section{ALGUNAS CONCLUSIONES Y RETOS}

En las páginas precedentes se ha tratado de sintetizar los elementos clave de la situación de desigualdad de mujeres y hombres en el empleo público. El presente trabajo lanza una serie de propuestas preliminares, algunas de las cuales han sido ya implementadas en otros países con cierto éxito, con el objetivo de abrir el debate a un mayor número de posibilidades en lo que a la construcción de sector público igualitario se refiere. Asimismo, consideramos conveniente realizar, en último lugar, unas puntualizaciones finales relativas a la situación actual y futura del empleo público que deben ser tenidas en cuenta en la adopción de cualquier política referida a este ámbito.

Nos encontramos con un sector público envejecido en el que, en los últimos años, se ha ido reduciendo el acceso a los puestos para los que se requiere un mayor nivel de formación (profesionales, científicos y técnicos), y que, por tanto, se encuentran mejor valorados y cuya provisión se realiza por medio pruebas de acceso objetivas no vinculadas a puestos concretos, lo que facilita el acceso de más mujeres (De Luxán Meléndez, 2018: 289, 302). Teniendo en cuenta el incremento de la presencia femenina en las universidades españolas, el rejuvenecimiento de aquellos puestos de trabajo del sector público que requieren estar en posesión de un título universitario, en principio debería contribuir a aumentar la presencia de mujeres en aquellos escalones de la Administración y el sector público en general en los que la remuneración percibida es mayor. Ahora bien, para que esta situación derive en una mejora de las condiciones de las mujeres en el sector público, es necesario que aquellas plazas que en los próximos años se irán vaciando por jubilación no desaparezcan o sean cubiertas en régimen de interinidad.

Otro elemento al que necesariamente debe prestarse atención es el riesgo que la creciente automatización de procesos y auge de la Administración electrónica puede tener para el trabajo de las mujeres en el sector público. Por una parte, la informatización de todo tipo de procesos conllevará la desaparición de determinados puestos de trabajo de secretaría y administración que, 
en la actualidad, son ejercidos en su mayoría por mujeres. Por otra parte, esta evolución también provoca una creciente demanda de personal especializado en informática y otras áreas del conocimiento similares en las que la presencia de mujeres es escasa.

Asimismo, ya desde hace tiempo se reclama por gran parte de la doctrina y se ha reconocido por las propias instituciones la necesidad de reformar los sistemas de acceso al empleo público (Ramió y Salvador, 2018). Encontramos aquí una importante disyuntiva entre aquellas voces que reclaman, no sin razón, una mayor flexibilización de los sistemas de acceso al empleo público en España (Castillo Blanco, 2020) y el nada desdeñable riesgo de que dicha flexibilización termine por perjudicar a las mujeres si no es correctamente abordada. Este es un elemento que, sin duda, debe tenerse en cuenta en la reforma de los sistemas de acceso y promoción, pero también, en general, de organización del empleo público.

Finalmente, en el actual contexto de pandemia mundial, cuyos efectos económicos negativos solo empiezan a vislumbrarse, es de gran relevancia que los poderes públicos y las y los representantes de la ciudadanía en las instituciones políticas sean conscientes de la gran importancia que tiene el empleo público como instrumento para la consecución de la igualdad de mujeres y hombres. Debe evitarse, por tanto, imitar los patrones de la anterior crisis económica y la contracción y precarización del empleo en el sector público que las medidas adoptadas entonces conllevaron.

\section{Bibliografía}

Alfama Guillén, E. (2015). Género, poder y Administraciones públicas: sobre la (im)posibilidad del cambio hacia una mayor igualdad. Una revisión de la literatura. Revista Española de Ciencia Política, 39, 263-287.

ANECA (2020). Resoluciones de sexenios de transferencia plenos de la CNEAI de diciembre de 2019, abril de 2020 y mayo de 2020. Disponible en: https://bit. ly/3tYDbQ2.

Ballester Pastor, M. A. (2019). El RDL 6/2019 para la garantía de la igualdad de trato y de oportunidades entre mujeres y hombres en el empleo y la ocupación: Dios y el diablo en la tierra del sol. Femeris, 4 (2), 14-38. Disponible en: https://doi. org/10.20318/femeris.2019.4763.

Bettio, F. y Verashchagina, A. (2009). Gender segregation in the labour market: Root causes, implications and policy responses in the EU. Luxembourg: OPOCE.

Blau, F. D. y Kahn, L. M. (2013). Female labour supply: Why is the United States falling behind? American Economic Review: Papers and Proceedings, 103 (3), 251-256. 
Boix Palop, A. y Soriano Arnanz, A. (2020). El acceso al empleo público en España: ejemplos comparados y propuestas de mejora. Valencia: Publicacions de la Universitat de València.

Butler, E. K., Winfree Jr, L. T. y Newbold, G. (2003). Policing and gender: male and female perspectives among members of the New Zealand Police. Police Quaterly, 6 (3), 298-329.

Cain Miller, C. (2015). When family-friendly policies backfire, The New York Times, 26-5-2015.

Carretero García, M. J. (2019). Brecha y discriminación salarial en las Administraciones Públicas: aspectos clave para su detección e intervención. Madrid: Fundación Mujeres. Disponible en: https://bit.ly/3nxZqKj.

Castillo Blanco, F. (2020). Un sistema de empleo público distópico: ¿sería preciso reformar el EBEP? Documentación Administrativa, 7, 8-32. Disponible en: https://doi.org/10.24965/da.i7.10889.

Charles, M. (2003). Deciphering sex segregation: Vertical and horizontal inequalities in ten national labor markets. Acta Sociologica, 46 (4), 267-287. Disponible en: https://doi.org/10.1177/0001699303464001.

Chen, R. H. (2015). Women in Policing: In Relation to Female Police Officers' Level of Motivation Toward the Career, Level of Stress, and Attitude of Misconduct. Sociological Imagination: Western's Undergraduate Sociology Student Journal, 4 (1), 1-17.

Cobo, R. (1995). Fundamentos del patriarcado moderno. Jean Jacques Rousseau. Madrid: Cátedra.

Comissió d'Igualtat de la Conselleria de Sanitat Universal i Salut Pública (2016). I Pla d'igualtat: 2016-2019. Valencia: Generalitat Valenciana. Disponible en: https://bit.ly/2QJtIh3.

Connell, R. (2006). Glass ceilings or gendered institutions? Mapping the gender regimes of public sector worksites. Public Administration Review, 66 (6), 837-849. Disponible en: https://doi.org/10.1111/j.1540-6210.2006.00652.x.

De Luxán Meléndez, J. M. (2018). Brecha salarial o discriminación laboral. Retribuciones y carrera profesional de las mujeres en el sector público. Derecho de las Relaciones Laborales, 3, 287-304.

De Luxán Meléndez, J. M. (2020). La estacionalidad en el empleo público desde una perspectiva de género. Un análisis de la estadística de afiliación de la seguridad social: 2014-2019. IgualdadES, 3, 473-517. Disponible en: https://doi. org/10.18042/cepc/IgdES.3.08.

Domínguez Martín y Sánchez-Sánchez. (2007). Los diferenciales salariales por género en España durante el desarrollismo franquista. Revista de Investigaciones Sociológicas, 117, 143-160. Disponible en: https://doi.org/10.2307/40184756.

EIGE (2020). Gender Equality Index. Disponible en: https://bit.ly/3eJSyFP.

Espinosa, J. y Bustelo, M. (2019). ¿Cómo evaluamos el éxito de las políticas de igualdad de género? Criterios y herramientas metodológicas. Revista Española de Ciencia Politica, 49, 151-172. Disponible en: https://doi.org/10.21308/ recp.49.07. 
Fernández de Castro, P. y Díaz García, O. (2016). La corresponsabilidad de género en las políticas de conciliación. En D. Carbonero Muñoz, E. Raya Díez, N. Caparrós Civera y C. Gimeno Monterde (coords.). Respuestas transdisciplinares en una sociedad global: aportaciones desde el trabajo social. Logroño: Universidad de la Rioja. Disponible en: https://bit.ly/3u53aFo.

Fernández García, C. M., Hernández García, J. y Rodríguez Pérez, S. (2014). Género y preferencias profesionales en universitarios de estudios científico-tecnológicos. Revista Española de Orientación y Psicopedagogía, 25 (1), 78-93.

Fernández Ruíz-Gálvez, E. (2003). Igualdad y Derechos Humanos. Madrid: Tecnos.

FESP-UGT (2021). Administraciones públicas. Brecha salarial en tiempos de la COVID19. Madrid: FeSP-UGT. Disponible en: https://bit.ly/3aJacbt.

González López, J. J. (2019). Abuso del empleo público temporal: respuestas administrativas y jurisdiccionales. Revista de Derecho UNED, 25, 61-88. Disponible en: https://doi.org/10.5944/rduned.25.2019.26986.

Hernández Martín, A., García-Valcárcel Muñoz-Repiso, A. y Sánchez Gómez, M. C. (2004). El profesorado universitario desde una perspectiva de género. Revista Educación, 28 (2), 105-143. Disponible en: https://doi.org/10.15517/revedu. v28i2.2256.

Instituto de las Mujeres (2019). Otros observatorios. Disponible en: https:/bit. ly/2PwM1Wb.

Instituto de las Mujeres (2020a). Igualdad en la empresa: preguntas frecuentes. Disponible en: https://bit.ly/3gURvoS.

Instituto de las Mujeres (2020b). Mujeres en cifras. Poder y toma de decisiones. Altos cargos de la Administración. Disponible en: https://bit.ly/3t4Cdk1.

INE (Instituto Nacional de Estadística) (2019). Encuesta de Población Activa. Segundo Trimestre de 2019. Ocupados a tiempo parcial por motivo de la jornada parcial, sexo y grupo de edad. Disponible en: https://cutt.ly/9ne2vxE.

López Díaz, A. J., Pereira, M. D., Dema Fernández, S. y Díaz Martínez, C. (2020). Encuesta AMIT sobre el sexenio de transferencia. Resultados preliminares. Toledo: AMIT. Disponible en: https://bit.ly/3vrXNR1.

Lousada Arochena, J. F. (2018). La igualdad retributiva de mujeres y hombres en la acción de Naciones Unidas y la Organización Internacional del Trabajo. Documentación Laboral, 3 (115), 33-51.

Mahoney, J. y Thelen, K. A. (2010). Explaining institutional change: Ambiguity, agency, and power. New York: Cambridge University Press. Disponible en: https://doi. org/10.1017/CBO9780511806414.

Maqueda, A. (2021). La Unión Europea presiona a España para bajar la temporalidad en la Administración. El País, 04-03-2021. Disponible en: https://bit. ly/3eDtH6h.

Marcos, L. (2018). Sanidad: el sector más feminizado con una brecha de 9000 euros al año en la pública. Cadena Ser, 01-03-2018. Disponible en: https://bit. ly/2Ranreo.

Marrades Puig, A. (2020). Diseñando un nuevo modelo económico: propuestas desde el derecho constitucional y la economía feminista sobre el cuidado y la 
igualdad frente a la crisis COVID-19. IgualdadES 3, 379-402. Disponible en: https://doi.org/10.18042/cepc/IgdES.3.04.

Martínez García, M. L. (2017). Maternidad y discriminación de las mujeres en el empleo. Anales de Derecho, 35 (2), 1-31.

Millán Vázquez de la Torre, M. G., Del Pilar Santos Pita, M. y Pérez Naranjo, L. (2015). Análisis del mercado laboral femenino en España: evolución y factores socioeconómicos determinantes del empleo. Papeles de Población, 21 (84), $197-225$.

Ministerio de Administraciones Públicas (2007). Boletín del Personal al Servicio de las Administraciones Públicas. Enero 2007. Madrid: Ministerio de Administraciones Públicas. Disponible en: https://bit.ly/2QJzfUO.

Ministerio de Hacienda y Función Pública (MHFP) (2018). Informe sobre la aplicación de politicas de igualdad: ejercicio 2017. Disponible en: https://bit.ly/3xsbMbv.

Ministerio de Sanidad (2009). Informe sobre profesionales de cuidados de enfermería. Oferta. Necesidad 2010-2025. Disponible en: https://bit.ly/2QF2l7W.

Ministerio de Política Territorial y Función Pública (MPTFP) (2020a). Boletín del personal al servicio de las Administraciones Públicas. Julio 2020. Madrid: Ministerio de Función Pública y Política Territorial. Disponible en: https://cutt.ly/ wntlKq6.

Ministerio de Política Territorial y Función Pública (MPTFP) (2020b). III Plan para la Igualdad de Género en la Administración General del Estado y en los organismos públicos vinculados o dependientes de ella. BOE, 29-12-2020. Disponible en: https://bit.ly/3nuQTrD.

Mora Villarrubia, R. y Ruíz-Castillo Ucelay, J. (2004). Gender segregation by occupations in the public and the private sector: The case of Spain. Investigaciones Económicas, 28 (3), 399-428.

Mosteiro García, M. J. (1997). El género como factor condicionante de la elección de carrera: hacia una orientación para la igualdad de oportunidades entre los sexos. Revista galego-portuguesa de psicoloxía e educación, 1, 305-315.

OCDE (2014). Women, government and policy making: fostering diversity for inclusive growth. Paris: OECD Publishing. Disponible en: https:/doi. org/10.1787/9789264210745-en.

OCDE (2019). Government at a glance 2019. Paris: OECD Publishing. Disponible en: https://bit.ly/2R6GhTI.

Oelz, M., Olney, S. y Tomei, M. (2013). Igualdad salarial: guia introductoria. Ginebra: Organización Internacional del Trabajo. Disponible en: https://bit.ly/3vzhSVP.

Organización Internacional del Trabajo (OIT) (2015). Igualdad de remuneración. Activador fundamental de la igualdad de género. Ginebra: Organización Internacional del Trabajo. Disponible en: https://bit.ly/3gO9OfB.

Paleo, N. y Alonso, A. (2014). ¿Es solo una cuestión de austeridad? Crisis económica y políticas de género en España. Investigaciones Feministas, 5, 36-68. Disponible en: https://doi.org/10.5209/rev_INFE.2014.v5.47987.

Pastor Gosálbez, P., Acosta Sarmiento, A., Torres Coronas, T. y Calvo Merino, M. (2020). Los planes de igualdad en las universidades españolas. Situación actual 
y retos de futuro. Educación XXI, 23 (1), 147-172. Disponible en: https://doi. org/10.5944/educxx 1.23873 .

Pateman, C. (1988). The sexual contract. Stanford: Stanford University Press.

PNUD (Programa de las Naciones Unidas para el Desarrollo) (2020). Gender Inequality Index (GII). Disponible en: https://bit.ly/2R3gJqp.

Ramió, C. y Salvador, M. (2018). La nueva gestión del empleo público: recursos humanos e innovación de la Administración. Barcelona: Tibidabo Ediciones.

Rodríguez Ruiz, B. y Rubio Marín, R. (2007). De la paridad, la igualdad y la representación en el estado democrático. Revista Española de Derecho Constitucional, 81, 115-159.

Rodríguez Ruiz, B. y Rubio Marín, R. (2009). Constitutional Justification of Parity Democracy. Alabama Law Review, 60 (5), 1171-1195.

Ruiloba Núñez, J. M. y Navarro González, R. (2020). ¿Administraciones públicas "genderizadas»? Revista Derecho del Estado, 47, 343-369. Disponible en: https:// doi.org/10.18601/01229893.n47.11.

Salazar Benítez, O. (2019). Democracia paritaria y Estado constitucional: de las cuotas a la ciudadanía radicalmente democrática. IgualdadES, 1, 43-81. Disponible en: https://doi.org/10.18042/cepc/IgdES.1.02.

Sánchez-Mira, N. (2017). La brecha salarial y las desigualdades de género en el mercado de trabajo. Una revisión de aproximaciones teóricas y aportaciones empíricas. Anuario IET de Trabajo y Relaciones Laborales, 4, 87-97. Disponible en: https://doi.org/10.5565/rev/aiet.56.

Santamaría Pastor, J. A. (2016). Principios de Derecho Administrativo General I. Madrid: Iustel.

Sevilla Merino, J., Belando Garín, B., Fabregat Monfort, G. y García Campá, S. (2017). Libro violeta de la función pública valenciana. Valencia: Servei de Publicacions de la Generalitat Valenciana.

Simpson, R. (2004). Masculinity at work: The experiences of men in female dominated occupations. Work, Employment and Society, 18 (2), 349-368. Disponible en: https://doi.org/10.1177/09500172004042773.

Tello-Sánchez, F. (2020). Empezar por casa: enfocando los planes de igualdad en la cultura organizacional de las administraciones públicas. Revista Vasca de Gestión de Personas y Organizaciones Públicas, 18, 114-135. Disponible en: https://doi. org/10.47623/ivap-rvgp.18.2020.06.

Tur Ausina, R. (2008). Título V: El principio de igualdad en el empleo público. Capítulo I: Criterios de actuación de las administraciones públicas. En J. I. García Ninet (dir.). Comentarios a la Ley de Igualdad (pp. 412-420). Bilbao: Wolters Kluwer.

UK Government (2019). Actions to close the gender pay gap. Disponible en: https:// bit.ly/3vGcQqf.

Vega Catena, P. J. (2014). La diferencia salarial por sexo en España. Un análisis a partir de la Encuesta de Estructura Salarial 2006 [tesis doctoral]. Universidad Rey Juan Carlos. Disponible en: https://bit.ly/3nxlWTp. 